\title{
Pharmacological treatment of hyperglycemia in type 2 diabetes
}

\author{
Simeon I. Taylor, Zhinous Shahidzadeh Yazdi, and Amber L. Beitelshees \\ Division of Endocrinology, Diabetes, and Nutrition, Department of Medicine, University of Maryland School of Medicine, Baltimore, Maryland, USA
}

\begin{abstract}
Diabetes mellitus is a major public health problem, affecting about $10 \%$ of the population. Pharmacotherapy aims to protect against microvascular complications, including blindness, end-stage kidney disease, and amputations. Landmark clinical trials have demonstrated that intensive glycemic control slows progression of microvascular complications (retinopathy, nephropathy, and neuropathy). Long-term follow-up has demonstrated that intensive glycemic control also decreases risk of macrovascular disease, albeit rigorous evidence of macrovascular benefit did not emerge for over a decade. The US FDA's recent requirement for dedicated cardiovascular outcome trials ushered in a golden age for understanding the clinical profiles of new type 2 diabetes drugs. Some clinical trials with sodium-glucose cotransporter-2 (SCLT2) inhibitors and glucagon-like peptide 1 (GLP1) receptor agonists reported data demonstrating cardiovascular benefit (decreased risk of major adverse cardiovascular events and hospitalization for heart failure) and slower progression of diabetic kidney disease. This Review discusses current guidelines for use of the 12 classes of drugs approved to promote glycemic control in patients with type 2 diabetes. The Review also anticipates future developments with potential to improve the standard of care: availability of generic dipeptidylpeptidase-4 (DPP4) inhibitors and SCLT2 inhibitors; precision medicine to identify the best drugs for individual patients; and new therapies to protect against chronic complications of diabetes.
\end{abstract}

Diabetes mellitus is a major public health problem, affecting about $10 \%$ of the population (1). Chronic complications of diabetes cause enormous human suffering, including blindness, kidney failure, amputations, myocardial infarction, and stroke. Inspired by the desire to develop better therapies, many researchers have investigated the pathophysiology of type 2 diabetes (T2D). While type 1 diabetes (T1D) is caused by autoimmune destruction of insulin-secreting $\beta$ cells of the pancreas, T2D is often associated with obesity and is characterized by both impaired insulin secretion and insulin resistance (2). T2D is a progressive disease. Insulin resistance manifests early in the natural history prior to occurrence of overt hyperglycemia. So long as pancreatic $\beta$ cells secrete sufficient insulin to compensate for insulin resistance, glucose levels are maintained at relatively normal levels (3). Overt diabetes occurs when $\beta$ cells no longer secrete sufficient insulin to maintain normoglycemia. Fasting hyperglycemia is driven by increased hepatic glucose production due to relatively low insulin levels combined with hepatic insulin resistance. Severity of metabolic defects increases over time, primarily because of increasingly severe impairment in insulin secretion.

This Review will discuss the state of the art in pharmacotherapy of T2D. Treatment aims to prevent or delay occurrence

Conflict of interest: SIT serves as a paid consultant for lonis Pharmaceuticals; was previously employed by Bristol Myers Squibb, where he contributed to R\&D on saxagliptin and dapagliflozin; and is an inventor on patents covering (a) the use of metreleptin as a treatment for lipodystrophy (US8318666B2) and (b) the use of fusion proteins containing fibronectin-derived serum albumin binding domains to extend the pharmacokinetics of therapeutic proteins (US9540424B2)

Copyright: (c) 2021, American Society for Clinical Investigation.

Reference information: J Clin Invest. 2021;131(2):e142243.

https://doi.org/10.1172/JCl142243. of microvascular and macrovascular complications - the main causes of morbidity and mortality in T2D. We focus specifically on hemoglobin $\mathrm{A}_{1 \mathrm{c}}$-lowering (HbA1c-lowering) drugs, although antihypertensives, lipid-lowering drugs, optimal nutrition, and physical exercise also contribute to a holistic approach to treatment.

\section{Therapeutic strategies and therapeutic targets for glycemic control}

The Diabetes Control and Complications Trial (DCCT) (4) demonstrated that enhanced glycemic control decreased risk of chronic microvascular complications. Patients with T1D were randomized between conventional insulin treatment and intensive insulin therapy, which sustained mean levels of glycated hemoglobin (HbA1c) at about 9\% and about 7\%, respectively, over a 10-year period. Patients receiving intensive insulin therapy experienced fewer microvascular complications. In the secondary prevention groups, intensive insulin therapy slowed progression of diabetic retinopathy by $54 \%$ and decreased risk of developing macroalbuminuria by $56 \%$. Two caveats must be added: First, there was a 3- to 5-year time lag before benefit was observed. Second, intensive insulin therapy increased the risk of serious hypoglycemia 3 -fold. A therapeutic target of $7.0 \%$ for $\mathrm{HbA1c}$ has been proposed to provide optimal balance between protection from microvascular complications and risk of serious hypoglycemia. Nevertheless, further decreases in $\mathrm{HbA1c}$ to levels below $7.0 \%$ were associated with further decreases in retinopathy progression. Moreover, in the Epidemiology of Interventions and Complications (EDIC) study, patients receiving intensive insulin therapy during DCCT experienced 57\% fewer major adverse cardiovascular events (MACE-3: death, nonfatal myocardial infarction, or nonfatal stroke) after 11 years of follow-up (5). 
The United Kingdom Prospective Diabetes Study (UKPDS) demonstrated that enhanced glycemic control is beneficial in T2D. T2D patients were randomized between conventional treatment (diet) and insulin or sulfonylurea for 10 years (6). Patients receiving either insulin or sulfonylureas had lower HbA1c levels (7.0\% vs. $7.9 \%$ ) and experienced $12 \%$ fewer diabetes-related endpoints, primarily a $25 \%$ decrease in microvascular endpoints. Both insulin and sulfonylureas were associated with increased weight gain and increased incidence of serious hypoglycemia. Overweight patients were randomized between diet and metformin (7). Patients receiving metformin had lower HbA1c levels (7.4\% vs. $8.0 \%$ ) and experienced $32 \%-36 \%$ lower risk of any diabetes-related endpoint, myocardial infarction, and all-cause mortality. Although treatment-associated differences in HbA1c disappeared during the first year of post-trial follow-up (8), risk reductions persisted for 10 years after UKPDS for patients treated with sulfonylureas or insulin: microvascular disease $(-24 \%)$, myocardial infarction (-15\%), and all-cause mortality $(-13 \%)$. In metformin-treated patients, significant risk reductions persisted for any diabetes-related endpoint $(-21 \%)$, myocardial infarction $(-33 \%)$, and death from any cause $(-27 \%)$.

\section{Subsequent studies investigated whether lower HbA1c targets provide protection against cardiovascular complications of T2D:}

ACCORD trial. In the Action to Control Cardiovascular Risk in Diabetes (ACCORD) trial (9), T2D patients with high cardiovascular risk were randomized between standard therapy (targeting HbA1c of 7.0\%-7.9\%) and intensive therapy (targeting HbA1c $<6.0 \%$ ). Therapeutic regimens were individualized at the investigators' discretion and aligned to each study group's HbA1c target. At 1 year, standard therapy achieved mean HbA1c of 7.5\%, while intensive therapy fell short of its objective of $<6.0 \%$ with an actual mean HbA1c of $6.4 \%$. The observation of higher mortality in the intensive-therapy group led to discontinuation of the study after a mean follow-up of 3.5 years (hazard ratio, 1.22; 95\% CI, 1.01-1.46). The primary outcome was first occurrence of nonfatal myocardial infarction, nonfatal stroke, or cardiovascular death (MACE-3). Kaplan-Meier curves began to diverge after 4 years with a $10 \%$ decrease in risk of MACE-3 at 6 years $(P=0.16)$. It is possible that intensive therapy would have demonstrated a statistically significant beneficial impact on MACE-3 had the study not been terminated early. Although the first publication speculated that increased risk of hypoglycemia might have increased all-cause mortality (9), later publications noted that high baseline levels of HbA1c were associated with high risk of death $(10,11)$. Nevertheless, intensive therapy exerted a strong protective effect to slow progression of diabetic retinopathy in ACCORD (adjusted odds ratio, 0.67 ; 95\% CI, 0.51-0.87) (12).

ADVANCE trial. In the Action in Diabetes and Vascular Disease (ADVANCE) trial (13), T2D patients with high risk of cardiovascular disease were randomized to standard therapy (HbA1c targets based on local guidelines) versus intensive control (targeting HbA1c $<6.5 \%$ ). After median follow-up of 5 years, standard and intensive therapy achieved mean HbA1c of $7.3 \%$ and $6.5 \%$, respectively. Intensive control decreased risk of a compos- ite outcome of major macrovascular plus microvascular adverse events (hazard ratio, $0.90 ; 95 \% \mathrm{CI}, 0.82-0.98$ ), primarily owing to decreased incidence of nephropathy. Intensive glucose control did not significantly affect risks of macrovascular endpoints. Nevertheless, because curves began to diverge after about 54 months, a beneficial impact on macrovascular outcomes might have become apparent had the study been continued longer.

VADT trial. In the Veterans Affairs Diabetes Trial (VADT) (14), T2D patients were randomized between standard and intensive treatment. Patients were started on metformin plus rosiglitazone (patients with $\mathrm{BMI} \geq 27$ ) or glimepiride plus rosiglitazone (BMI <27). Intensive-therapy patients were started on maximal doses, whereas standard-therapy patients were started on half-maximal doses. Insulin was added if intensive-therapy group patients did not achieve $\mathrm{HbA1c}<6 \%$ and if standard-therapy group patients had HbA1c $\geq 9 \%$. Mean levels of $\mathrm{HbA1c}$ were $8.4 \%$ and $6.9 \%$ for the standard- and intensive-therapy groups, respectively. There was a statistically insignificant trend toward decreased risk in the intensivetherapy group (hazard ratio, 0.88) for the primary outcome (a composite of seven macrovascular disease endpoints).

What can be concluded on the basis of these studies?

Microvascular complications. DCCT $(4,5)$ and UKPDS (6-8) demonstrated that enhanced glycemic control slows progression of microvascular complications (retinopathy, nephropathy, and neuropathy). Outcome measures were limited to biomarkers such as retinal photographs rather than hard endpoints such as blindness. Pharmacotherapy was limited to insulin, metformin, or sulfonylureas. Both clinical trials established HbA1c as a surrogate biomarker for therapeutic benefit and helped to define HbA1c targets to guide therapy. ACCORD (9) and ADVANCE (13) confirmed that intensive glycemic control slowed progression of microvascular complications.

Macrovascular complications. Long-term follow up in DCCT/ EDIC $(4,5)$ and UKPDS $(6-8)$ strongly supports the conclusion that intensive glycemic control can - in the fullness of time - improve cardiovascular outcomes. It is not clear whether cardioprotection is a direct consequence of enhanced glycemic control or is mediated indirectly through beneficial effects on renal function. In any case, the beneficial impact on cardiovascular outcomes was not rigorously demonstrated during the first 10 years, but became apparent during long-term follow up after interventions had ended $(5,8)$. Importantly, a trend toward cardiovascular benefit began to emerge in ACCORD and ADVANCE only after $48-54$ months of intensive glycemic control $(9,13)$. A similar lag was noted with statins - a drug class that is well recognized to decrease the risk of major adverse cardiovascular events. Kaplan-Meier curves for diabetic patients in the Scandinavian Simvastatin Survival Study were superimposable during the first 2 years, but a clear beneficial effect emerged during the ensuing 4 years of simvastatin therapy (15). Thus, ACCORD, ADVANCE, and VADT were likely too short to adequately test the hypothesis that enhanced glycemic control provides cardiovascular benefit. Furthermore, those studies were conducted before widespread availability of newer drugs such as glucagon-like peptide 1 receptor agonists (GLP1RAs), sodium-glucose cotransporter-2 inhibitors (SGLT2is), and dipeptidylpeptidase-4 inhibitors (DPP4is). 


\section{Table 1. Twelve classes of drugs approved in the United States to decrease HbA1c in patients with T2D}

\begin{tabular}{|c|c|c|c|c|c|}
\hline Drug class & Approved drugs (US) & $\begin{array}{l}\text { Baseline } \\
\text { HbA1c }\end{array}$ & $\Delta$ HbA1c (\%) & Selected safety issues & Comments \\
\hline Biguanides & $\begin{array}{c}\text { Metformin } \mathrm{HCl} \\
\text { Metformin extended release }\end{array}$ & $\begin{array}{l}8.4 \% \\
8.4 \%\end{array}$ & $\begin{array}{l}\text { Met-HCl: }-1.8 \% \text { (titrated dose) } \\
\text { Met-XR: }-1.0 \% \text { (2000 mg/d) }\end{array}$ & $\begin{array}{l}\text { Lactic acidosis; vitamin } \mathrm{B}_{12} \\
\text { deficiency; abdominal pain, } \\
\text { diarrhea, nausea }\end{array}$ & $\begin{array}{l}\text { Placebo-subtracted monotherapy. } \\
\text { Sources: metformin HCI (92); } \\
\text { metformin XR (93). }\end{array}$ \\
\hline Sulfonylureas & $\begin{array}{c}\text { Climepiride } \\
\text { Clipizide } \\
\text { Clibenclamide (glyburide) }\end{array}$ & $\begin{array}{l}7.7 \% \\
7.6 \%\end{array}$ & $\begin{array}{l}\text { Climepiride (mean, } 3 \mathrm{mg} / \mathrm{d}):-0.6 \% \\
\quad \text { Clipizide }(5-20 \mathrm{mg} / \mathrm{d}):-0.6 \%\end{array}$ & $\begin{array}{c}\text { Hypoglycemia; weight gain; } \\
\text { potential increased risk of CV } \\
\text { mortality }\end{array}$ & $\begin{array}{l}\text { HbA1c-lowering from baseline in } \\
\text { patients inadequately controlled on } \\
\text { metformin. Climepiride data from PI } \\
\text { for linagliptin. Clipizide data from PI } \\
\text { for sitagliptin. }\end{array}$ \\
\hline Thiazolidinediones & $\begin{array}{l}\text { Pioglitazone } \\
\text { Rosiglitazone }\end{array}$ & $\begin{array}{l}9.9 \% \\
8.9 \% \\
8.9 \%\end{array}$ & $\begin{array}{l}\text { Pioglitazone }(30 \mathrm{mg} / \mathrm{d}):-0.8 \% \\
\text { Rosiglitazone }(4 \mathrm{mg} / \mathrm{d}):-1.0 \% \\
\text { Rosiglitazone }(8 \mathrm{mg} / \mathrm{d}):-1.2 \%\end{array}$ & $\begin{array}{l}\text { Peripheral edema; congestive } \\
\text { heart failure; weight gain; bone } \\
\text { fractures (esp. in females) }\end{array}$ & $\begin{array}{l}\text { Source: Pls for } 2 \text { drugs. } \\
\triangle H b A 1 c \text { : placebo-subtracted data. } \\
\text { PROactive suggested decreased risk } \\
\text { of MACE-3 for pioglitazone. }\end{array}$ \\
\hline $\begin{array}{l}\text { Dipeptidylpeptidase-4 } \\
\text { (DPP4) inhibitors }\end{array}$ & $\begin{array}{l}\text { Alogliptin } \\
\text { Linagliptin } \\
\text { Saxagliptin } \\
\text { Sitagliptin }\end{array}$ & $\begin{array}{l}8.6 \% \\
7.7 \% \\
8.1 \% \\
8.0 \%\end{array}$ & $\begin{array}{l}\text { Alogliptin ( } 25 \mathrm{mg} / \mathrm{d}):-0.9 \% \\
\text { Linagliptin ( } 5 \mathrm{mg} / \mathrm{d}):-0.4 \% \\
\text { Saxagliptin }(5 \mathrm{mg} / \mathrm{d}):-0.8 \% \\
\text { Sitagliptin (100 mg/d): }-0.7 \%\end{array}$ & $\begin{array}{l}\text { Angioedema (esp. with ACE } \\
\text { inhibitor); joint pain; } \\
\text { pancreatitis listed in } \\
\text { some PIs }\end{array}$ & $\begin{array}{l}\Delta \text { HbA1c: placebo-subtracted data } \\
\text { for saxagliptin; change from baseline } \\
\text { for other drugs. Studies conducted in } \\
\text { patients inadequately controlled on } \\
\text { metformin. Source: Pl for each drug. }\end{array}$ \\
\hline $\begin{array}{l}\text { Clucagon-like peptide } 1 \\
\text { (GLP1) receptor agonists }\end{array}$ & $\begin{array}{c}\text { Albiglutide } \\
\text { Dulaglutide } \\
\text { Exenatide ER } \\
\text { Liraglutide } \\
\text { Lixisenatide } \\
\text { Semaglutide (s.c. injection) } \\
\text { Semaglutide (oral) }\end{array}$ & $\begin{array}{l}8.1 \% \\
8.1 \% \\
8.6 \% \\
8.4 \% \\
7.95 \% \\
8.4 \% \\
8.1 \%\end{array}$ & $\begin{array}{l}\text { Albiglutide (30 mg/d): }-0.9 \% \\
\text { Dulaglutide }(1.5 \mathrm{mg} / \mathrm{wk}):-1.1 \% \\
\text { Exenatide ER }(2 \mathrm{mg} / \mathrm{wk}):-1.5 \% \\
\text { Liraglutide }(1.8 \mathrm{mg} / \mathrm{d}):-1.5 \% \\
\text { Lixisenatide }(10 \mu \mathrm{g} / \mathrm{d}):-0.73 \% \\
\text { Semaglutide }(1 \mathrm{mg} / \mathrm{wk}, \text { s.c.): }-1.4 \% \\
\text { Semaglutide }(14 \mathrm{mg} / \mathrm{d}, \text { p.0.): }-1.3 \%\end{array}$ & $\begin{array}{l}\text { Nausea and vomiting; PI for } \\
\text { some drugs lists pancreatitis; } \\
\text { contraindicated in case of } \\
\text { personal or familial history of } \\
\text { MTC or MEN2 }\end{array}$ & $\begin{array}{l}\text { HbA1c-lowering from baseline in } \\
\text { patients inadequately controlled } \\
\text { on metformin. Source: PI for each } \\
\text { drug. Clinical trials: decreased risk of } \\
\text { MACE-3 for liraglutide, dulaglutide, } \\
\text { semaglutide, and albiglutide. }\end{array}$ \\
\hline Insulins & $\begin{array}{l}\text { Rapid-acting insulins } \\
\text { Basal insulins }\end{array}$ & Various & Dose-dependent & Hypoglycemia; weight gain & $\begin{array}{l}\text { HbA1c-lowering depends on insulin } \\
\text { dose. }\end{array}$ \\
\hline \multirow[t]{2}{*}{ Meglitinides } & Repaglinide & $8.3 \%$ & Repaglinide (0.5-4 mg, tid): $-1.08 \%$ & Hypoglycemia & $\begin{array}{l}\text { HbA1c-lowering corrected for effect of } \\
\text { metformin monotherapy. Source: PI. }\end{array}$ \\
\hline & Nateglinide & $8.7 \%$ & Nateglinide (120 mg, tid): $-0.6 \%$ & & \\
\hline Amylinomimetic & Pramlintide & $9.0 \%$ & Pramlintide (120 $\mu \mathrm{g}$, tid): $-0.3 \%$ & $\begin{array}{c}\text { Hypoglycemia; contraindicated in } \\
\text { gastroparesis or } \\
\text { hypoglycemia unawareness }\end{array}$ & $\begin{array}{l}\text { HbA1c-lowering assessed relative } \\
\text { to the effects of background insulin } \\
\text { therapy. Source: PI. }\end{array}$ \\
\hline
\end{tabular}

Most data were obtained from FDA-approved prescribing information (PI). When sulfonylureas were approved, the PI did not report HbA1c-lowering; so efficacy data for glimepiride and glipizide were obtained from PI for linagliptin and sitagliptin, respectively. The table lists HbA1c-lowering for monotherapy with metformin $(92,93)$. For other drugs, the table lists efficacy data for second-line therapy - most often in patients who were inadequately controlled on metformin. ACE, angiotensin-converting enzyme; CV, cardiovascular; DKA, diabetic ketoacidosis; ER, extended release; MEN2, multiple endocrine neoplasia type 2; MTC, medullary thyroid cancer.

\section{Glucose-lowering drugs to treat $T 2 D$}

Twelve classes of drugs are approved to treat T2D: biguanides (e.g., metformin), sulfonylureas, thiazolidinediones (TZDs), DPP4is, SGLT2is, GLP1RAs, insulins, $\alpha$-glucosidase inhibitors, dopaminergic antagonists, bile acid sequestrants, meglitinides, and amylinomimetics (Table 1). The last five are less commonly used and will not be discussed extensively in this Review. Figure 1 briefly reviews mechanisms of action of commonly used classes of drugs. Sulfonylureas and biguanides were the only oral treatments for T2D from the 1950s until the mid-1990s. Two new classes of oral antidiabetic drugs were launched in the 1990s: $\alpha$-glucosidase inhibitors and PPAR $\gamma$ agonists. Additional classes of diabetes drugs were 


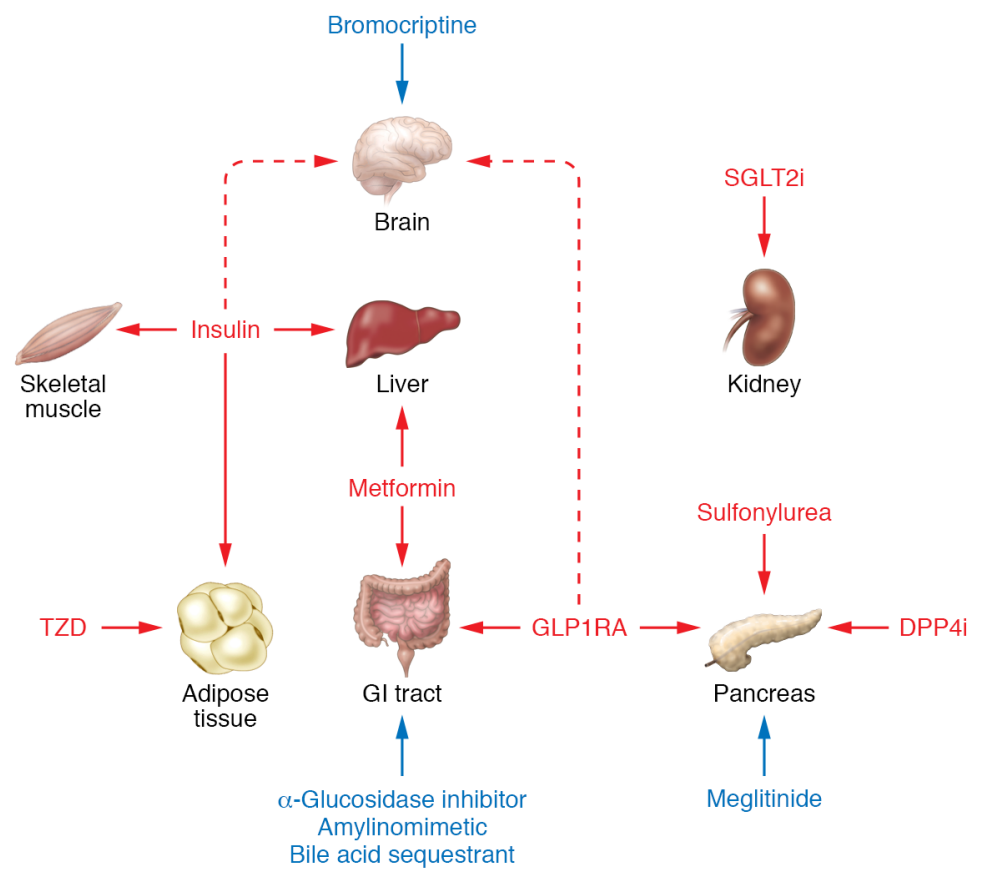

Figure 1. Mechanisms of action for drugs approved for glycemic control in patients with T2D. Seven classes of FDA-approved T2D drugs are widely used: Biguanides. Several hypotheses have been proposed for metformin's mechanism of action: (i) activation of AMPK $(102,103)$; (ii) inhibition of hepatic adenylyl cyclase (104); (iii) inhibition of mitochondrial glycerol-3-phosphate dehydrogenase (GPD2) (105); (iv) increasing levels of GDF15 (106, 107); and (v) alteration of intracellular protein-bound iron levels (108). Metformin decreases hepatic glucose production (109). Its action may be exerted in the gut independent of absorption into the circulation (110). Sulfonylureas bind to sulfonylurea receptor-1 (SUR-1) in pancreatic $\beta$ cells, leading to closure of the ATP-inhibitable $\mathrm{K}^{+}$channel $\mathrm{K}_{\mathrm{i}} 6.1$, thereby initiating electrophysiological changes that trigger insulin secretion (111). Thiazolidinediones (TZDs) activate PPAR $\gamma$, an adipose tissue transcription factor (112). TZDs promote triglyceride storage in adipose tissue while decreasing deposition of triglycerides in liver and muscle, thereby increasing insulin sensitivity $(113,114)$. GLP1RAs bind to the GLP1 receptor on pancreatic $\beta$ cells, augmenting glucose-stimulated insulin secretion. GLP1RAs also delay gastric emptying, decrease glucagon levels, and decrease food intake (115). DPP4is protect GLP1 from degradation, thereby potentiating endogenous GLP1 activity. SCLT2is promote glucosuria and natriuresis by inhibiting proximal tubular glucose reabsorption. Glucosuria decreases HbA1c levels and promotes weight loss. Natriuresis decreases blood pressure, and may also protect against congestive heart failure (41). Insulin binds to its receptor in liver, skeletal muscle, and adipose tissue, triggering intracellular signaling pathways that mediate the various biological actions of insulin - including increased glucose transport into skeletal muscle and adipocytes, regulation of several key metabolic enzymes, and regulation of gene expression (116). Five other classes of approved drugs are less widely used: meglitinides, $\alpha$-glucosidase inhibitors, amylinomimetics, bile acid sequestrants, and bromocriptine (a D2/D3 dopaminergic agonist).

introduced between 2005 and 2013, the most important being GLP1RAs, DPP4is, and SGLT2is.

Comparative effectiveness. Head-to-head trials have not compared efficacies of individual drugs (or specific drug regimens) with respect to "hard" clinical endpoints such as blindness, amputations, kidney failure, death, myocardial infarction, stroke, or heart failure. Most outcome trials have compared benefits of therapeutic strategies (e.g., targeting different levels of HbA1c) rather than efficacy of individual drugs. The ADOPT trial (A Diabetes Outcome Progression Trial) is an exception, comparing metformin, glyburide, and rosiglitazone with respect to time to secondary failure (16). Although rosiglitazone performed best with respect to ADOPT's primary outcome, TZDs are no longer widely used because of safety concerns about increased risk of heart failure and bone fracture (17-20). While glyburide performed worst, sulfonylureas continue to be widely prescribed (21), primarily because of their low cost. The NIH has designed a similar comparative effectiveness trial, Glycemia Reduction Approaches in Diabetes Effectiveness (GRADE; ClinicalTrials. gov NCT01794143), with time to "treatment failure" (HbA1c $\geq 7.0 \%)$ as its primary endpoint (22). GRADE compares four classes of drugs added to background therapy with metformin: sulfonylureas, insulin, DPP4is, and GLP1RAs - but not SGLT2is. The GRADE trial (5047 participants) is not powered to assess comparative effectiveness with respect to the most important clinical outcomes, such as blindness, end-stage kidney disease, amputations, or MACE-3. ClinicalTrials.gov lists the primary completion date as July 2021.

Data derived from different trials cannot be compared in a scientifically rigorous way. The magnitude of lowering of $\mathrm{HbA1C}$ is strongly correlated with baseline values of HbA1c (23) and tends to be larger in studies with higher HbA1c at baseline. Further, HbA1c-lowering may be different when a drug is used as monotherapy as compared with combination therapy. Late-stage patients with more severely impaired $\beta$ cell function may experience a lesser lowering of HbA1c, especially with drugs targeting $\beta$ cells. These critical factors often differ among clinical trials. Table 2 summarizes headto-head trials comparing HbA1c-lowering efficacy of individual drugs. Some head-to-head trials were conducted in the context of developing fixed-dose combination pills. Although head-to-head comparisons are informative, relatively few head-to-head trials have been conducted. "Real-world" epidemiological data are sometimes used to compare different classes of drugs (24), but efforts to correct for confounders may not fully account for systematic differences among the people receiving individual drugs (e.g., differences in sex, race, medical insurance, or socioeconomic situation). These considerations create major challenges for physicians and patients to develop evidencebased strategies.

Based on data derived from head-to-head clinical trials (Table 2), we offer a few conclusions: (a) In headto-head monotherapy studies comparing metformin (typically doses of $\sim 2000 \mathrm{mg} / \mathrm{d}$ ) with other drugs, metformin-induced decreases in HbA1c ranged from about $1.0 \%$ to about 1.8\%. Larger decreases were generally observed in trials with the highest baseline HbA1c levels. (b) HbA1c-lowering provided by sulfonylurea monotherapy was about $0.2 \%-0.3 \%$ greater than that observed with metformin - albeit this effect is not sustained over time. (c) HbA1c-lowering provided by monotherapy with TZDs or SGLT2is was comparable to that seen with metformin. (d) In head-to-head trials, DPP4i monotherapy was less effective than metformin. (e) In patients inadequately controlled on metformin, liraglutide $(1.8 \mathrm{mg} / \mathrm{d})$ provided greater HbA1c-lowering than sitagliptin (100 
Table 2. Head-to-head comparative effectiveness trials of drugs to treat T2D

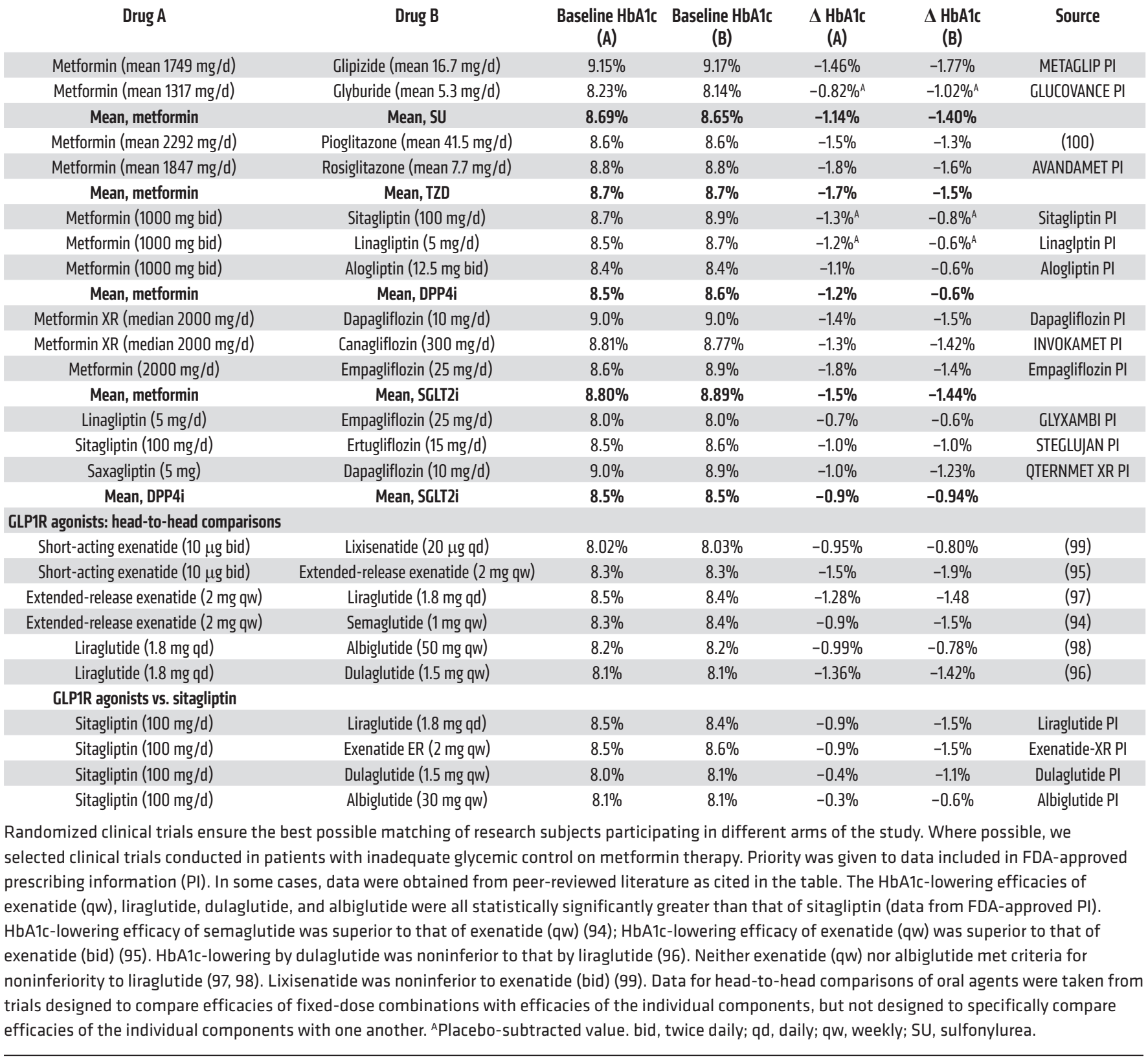

$\mathrm{mg} / \mathrm{d}$ ) $-1.5 \%$ versus $0.9 \%$, respectively (25). (f) Five head-to-head trials between GLP1RAs demonstrated that HbA1c-lowering efficacy varied widely, with the following estimates of "normalized HbA1c-lowering": lixisenatide, $-0.82 \%$; short-acting exenatide, $-1.0 \%$; albiglutide, $-1.16 \%$; long-acting exenatide, $-1.27 \%$; liraglutide, $-1.47 \%$; dulaglutide, $-1.53 \%$; and semaglutide, $-2.12 \%$ (26). (g) GLP1RAs (especially semaglutide) provide greater HbA1clowering than DPP4is or SGLT2is when added to regimens of patients inadequately controlled on metformin.

\section{Cardiovascular outcome trials}

In response to concerns that some $\mathrm{T} 2 \mathrm{D}$ drugs increase cardiovascular risks (27), the FDA requires sponsors to provide additional cardiovascular safety data: (a) a preapproval analysis to exclude an $80 \%$ increase in cardiovascular risk; and (b) a dedicated postapproval trial to exclude a $30 \%$ increase in risk (28). An FDA advisory committee recommended that clinical trials compare new T2D drugs to the standard of care and that patients in both treatment arms should be matched with respect to levels of HbA1c and known cardiovascular risk factors (e.g., blood pressure and LDL cholesterol). These recommendations were not followed in practice. In any case, the FDA's requirements ushered in a golden age of cardiovascular outcome trials (CVOTs) providing insights into GLP1RAs, DPP4is, and SGLT2is (Table 3). These clinical trials were not powered to provide rigorous comparisons among different subpopulations nor to correct for multiple comparisons 
Table 3. Summary of cardiovascular outcome trials of diabetes drugs, 2005-2020

\begin{tabular}{|c|c|c|c|c|c|c|c|c|c|}
\hline Drug & Study name & Inclusion criteria & $N$ & $\begin{array}{c}\text { Mean } \\
\text { duration }\end{array}$ & Comparator & $\begin{array}{c}\text { Baseline mean } \\
\text { HbA1c }\end{array}$ & $\begin{array}{l}\text { HR, MACE } \\
(95 \% \mathrm{Cl})\end{array}$ & $\begin{array}{c}P \text { value } \\
\text { (superiority) }\end{array}$ & References \\
\hline Pioglitazone & PROactive & Macrovascular disease & 5238 & $2.9 \mathrm{yr}$ & Placebo & $7.8 \% / 7.9 \%$ & $0.84(0.72-0.98)$ & 0.027 & 54 \\
\hline Rosiglitazone & RECORD & $\begin{array}{l}\text { Monotherapy with } \\
\text { metformin or SU }\end{array}$ & 4447 & $5.5 \mathrm{yr}$ & Sulfonylurea+metformin & $7.9 \% / 7.9 \%$ & $0.95(0.78-1.17)$ & -- & 18,19 \\
\hline Sitagliptin & TECOS & Established CV disease & 14,671 & $3.0 \mathrm{yr}$ & Placebo & $7.2 \%$ & 0.98 (0.89-1.08) & 0.65 & 33 \\
\hline Saxagliptin & SAVOR-TIMI 53 & $\begin{array}{c}\text { History of CV disease or } \\
\text { high CV risk }\end{array}$ & 16,492 & $2.1 \mathrm{yr}$ & Placebo & $8.0 \% / 8.0 \%$ & $1.00(0.89-1.12)$ & 0.99 & 31 \\
\hline Alogliptin & EXAMINE & Acute coronary syndrome & 5380 & $1.5 \mathrm{yr}$ & Placebo & $8.0 \% / 8.0 \%$ & $0.96(<1.16)$ & 0.32 & 32 \\
\hline Linagliptin & CAROLINA & High CV risk & 6033 & $6.3 \mathrm{yr}$ & Glimepiride & $7.2 \% / 7.2 \%$ & $0.98(0.84-1.14)$ & 0.76 & 34 \\
\hline Empagliflozin & EMPA-REG & Established CV disease & 7028 & $2.6 \mathrm{yr}$ & Placebo & $8.07 \% / 8.08 \%$ & $0.86(0.74-0.99)$ & 0.04 & 37 \\
\hline Canagliflozin & CANVAS & $\begin{array}{c}A S C V D \text { or } \geq 2 \text { CV risk } \\
\text { factors }\end{array}$ & 10,142 & $3.6 \mathrm{yr}$ & Placebo & $8.2 \% / 8.2 \%$ & $0.86(0.75-0.97)$ & 0.02 & 38 \\
\hline Ertugliflozin & VERTIS CV & Established CV disease & 8246 & $3.5 \mathrm{yr}$ & Placebo & $8.2 \% / 8.2 \%$ & $\begin{array}{c}0.91(5 \mathrm{mg})(0.77- \\
1.07) ; 1.04(15 \mathrm{mg}) \\
(0.89-1.21)\end{array}$ & -- & 40 \\
\hline Lixisenatide & ELIXA & Acute coronary syndrome & 6068 & $1.9 \mathrm{yr}$ & Placebo & $7.7 \% / 7.6 \%$ & $1.02(0.89-1.17)$ & 0.81 & 52 \\
\hline Liraglutide & LEADER & High CV risk & 9340 & $3.8 \mathrm{yr}$ & Placebo & $8.7 \% / 8.7 \%$ & $0.87(0.78-0.97)$ & 0.01 & 46 \\
\hline Semaglutide & SUSTAIN-6 & $\begin{array}{c}\text { Established CVD, CKD, } \\
\text { or CHF }\end{array}$ & 3297 & $2 \mathrm{yr}$ & Placebo & $8.7 \% / 8.7 \%$ & $0.74(0.58-0.95)$ & 0.02 & 47 \\
\hline Exenatide & EXSCEL & Prior CVD (73\%) & 14,752 & $3.2 \mathrm{yr}$ & Placebo & $8.1 \% / 8.1 \%$ & $0.91(0.83-1.00)$ & 0.06 & 51 \\
\hline Albiglutide & $\begin{array}{l}\text { HARMONY } \\
\text { OUTCOMES }\end{array}$ & History of CV disease & 9463 & $1.6 \mathrm{yr}$ & Placebo & $8.72 \% / 8.76 \%$ & $0.78(0.68-0.90)$ & 0.0006 & 50 \\
\hline Dulaglutide & REWIND & ASCVD or high CV risk & 9901 & $5.4 \mathrm{yr}$ & Placebo & $7.4 \% / 7.3 \%$ & $0.88(0.79-0.99)$ & 0.026 & 48 \\
\hline Bromocriptine & $\begin{array}{c}\text { Cycloset Safety } \\
\text { Trial }\end{array}$ & $\begin{array}{c}\text { T2D; stable Rx for } \geq 30 \\
\text { days }\end{array}$ & 3070 & $0.5-1 \mathrm{yr}$ & Placebo & $7.0 \% / 7.0 \%$ & $0.61(0.38-0.97)$ & -- & 101 \\
\hline
\end{tabular}

The table summarizes information on cardiovascular outcome trials evaluating the impact of individual glucose-lowering drugs on the risk of major adverse cardiovascular events. Several cardiovascular outcome trials were initiated before 2008 when the FDA began to require formal cardiovascular outcome trials for all T2D drugs: pioglitazone, rosiglitazone, and sitagliptin. For completeness, we have included the Cycloset Safety Trial of bromocriptine, despite challenges in interpreting the data in light of the low percentage of patients who completed the study ( $53 \%$ of bromocriptine-treated patients vs. $68 \%$ of placebo-treated patients). Moreover, many patients in Cycloset were lost to follow-up: 5.6\% (bromocriptine) and 5.6\% (placebo). The table presents hazard ratios based on data for the three-component composite for major adverse cardiovascular events (MACE-3): cardiovascular death, nonfatal myocardial infarction, and nonfatal stroke. In some cases, MACE-3 was not prespecified as the study's original primary outcome (PROactive and RECORD); in those cases, we have presented nominal $P$ values uncorrected for multiple comparisons. In the case of ELIXA, the table presents data on four-component MACE- 4 (components of MACE-3 plus hospitalization for unstable angina). However, because hospitalization for unstable angina represented $\leq 2.5 \%$ of the major adverse cardiovascular events in ELIXA, it is likely that the hazard ratio for MACE-3 would have been very similar. ASCVD, atherosclerotic cardiovascular disease; CHF, congestive heart failure; CKD, chronic kidney disease; CV, cardiovascular; CVD, cardiovascular disease; HR, hazard ratio; SU, sulfonylurea.

required for such comparisons. Nevertheless, it is noteworthy that meta-analyses have questioned whether Black people experienced the same benefit as the overall populations in studies of SGLT2is or GLP1RAs (29) and whether women experienced the same benefit as men in studies with SGLT2is (30). Although individuals can vary widely in their responses to specific drugs, the current state of the art focuses primarily on average patient responses, which does not account for interindividual variation nor variation among various subpopulations based on factors such as sex, race, or ethnicity.

DPP4is. Dedicated CVOTs reported hazard ratios between 0.96 and 1.00 for MACE-3 for all four DPP4is (Table 3) (27). Compared with placebo-treated patients, HbA1c levels were modestly lower $(20.3 \%)$ in patients receiving sitagliptin, saxagliptin, or alogliptin (31-33). Linagliptin- and glimepiride-treated patients had similar HbA1c levels (34). All four trials satisfied the FDA's primary objective by ruling out significant increases in cardiovascular risk. Previous meta-analyses of phase III data with sitagliptin and saxagliptin $(35,36)$ suggested that these drugs might protect against major adverse cardiovascular events, but dedicated CVOTs conducted in patients with higher cardiovascular risk did not confirm this prediction. Nevertheless, analyses of real-world data demonstrated that metformin+DPP4i treatment was associated with $23 \%-24 \%$ lower all-cause mortality and MACE-3 than metformin+sulfonylurea therapy (24). Interestingly, subgroup analysis suggested that improved outcomes were limited to people without high cardiovascular risk and were not observed in highrisk individuals (24).

SGLT2is. CVOTs for empagliflozin and canagliflozin $(37,38)$ both demonstrated $14 \%$ decreases in risk of MACE-3. In contrast, CVOTs with dapagliflozin and ertugliflozin failed to meet prespecified criteria for statistical significance $(39,40)$. A recent meta-analysis of all four SGLT2is estimated a hazard ratio of 0.90 (95\% CI, 0.85-0.95) for MACE-3 (40). It remains uncertain whether there are real and reproducible differences among the 
four SGLT2is or whether numerical differences between drugs resulted from random variation. Meta-analysis demonstrated a strong effect of SGLT2is to decrease the risk of hospitalization for heart failure, with a hazard ratio of 0.68 (95\% CI, 0.61-0.76) (40). Similarly, SGLT2is slowed progression of diabetic kidney disease, with meta-analysis estimating a hazard ratio of 0.62 (95\% CI, 0.56-070) (40). The heart failure and renoprotection benefits were quantitatively larger and more consistently observed than improvement in MACE-3 outcomes.

What mechanisms mediate improved outcomes due to SGLT2is? All trials demonstrated significantly lower levels of HbA1c and blood pressure, which may have contributed to improved outcomes (27). SGLT2is' natriuretic action may contribute to beneficial impact on heart failure (41). Finally, analysis of data from the EMPA-REG OUTCOME trial suggested that increased hematocrit may mediate decreased risk for cardiovascular mortality (42). Acutely, drug-induced natriuresis leads to volume contraction and hemoconcentration (41). However, SGLT2is also trigger increased erythropoiesis. Furthermore, renoprotective benefit may be mediated by inhibition of proximal tubular sodium reabsorption, which in turn (a) increases sodium delivery to the macula densa (43), (b) modulates tubuloglomerular feedback, (c) corrects glomerular hyperfiltration, and (d) slows progression of kidney disease. CVOTs were specifically designed to provide information about cardiovascular and kidney outcomes but also identified unexpected safety issues. The CANVAS study with canagliflozin demonstrated increased risk of lower-extremity amputations (38), accelerated loss of bone mineral density, and increased risk of fracture $(41,44,45)$.

GLP1RAs. Liraglutide, dulaglutide, semaglutide, and albiglutide were reported to decrease risk for MACE-3 (46-50). Approved doses of liraglutide, dulaglutide, and semaglutide provide the largest improvements in HbA1c among GLP1RAs (26), whereas extended-release exenatide (51) and lixisenatide (52) did not demonstrate noninferiority to liraglutide for HbA1c-lowering. Analysis of data from the LEADER trial with liraglutide suggested that HbA1c-lowering may have mediated about $83 \%$ of liraglutide's cardiovascular benefit (53). CVOTs for GLP1RAs were conducted as placebo-controlled studies; patients in placebo groups had substantially higher HbA1c levels than patients receiving GLP1RAs (26). Although cardiovascular risk decreased immediately in CVOTs with canagliflozin and empagliflozin (37, 38), about 1 year elapsed before onset of benefit with GLP1RAs. While liraglutide, dulaglutide, canagliflozin, and empagliflozin decreased the risk of MACE- 3 by $12 \%-14 \%(37,38,46,48)$, semaglutide provided numerically larger cardioprotection $(26 \%$ decreased risk of MACE-3) (47).

Thiazolidinediones. CVOTs were conducted for both pioglitazone $(20,54)$ and rosiglitazone $(18,19)$. The PROactive trial with pioglitazone resembled FDA-mandated CVOTs, with the exception that MACE-3 was prespecified as a secondary outcome rather than the primary outcome. Nevertheless, pioglitazone decreased risk of MACE-3 (hazard ratio, $0.84 ; 95 \% \mathrm{CI}, 0.72-0.98 ; P=0.027$ ). In contrast, RECORD compared rosiglitazone monotherapy with metformin or a sulfonylurea $(18,19)$. RECORD reported a hazard ratio of 0.95 (95\% CI, 0.78-1.17), ruling out a $30 \%$ increase in risk, as the FDA now requires.
Real-world data. While randomized controlled trials provide the highest level of scientific rigor, "real-world" epidemiological studies provide complementary information about comparative effectiveness and other topics not assessed in randomized clinical trials. Jensen et al. (24) analyzed real-world data from a cohort of 66,807 Danish people with T2D. When combined with metformin, DPP4is, SGLT2is, and GLP1RAs were associated with improved outcomes compared with dual therapy with metformin plus sulfonylurea: hazard ratios for severe hypoglycemia were 0.05-0.10; hazard ratios for MACE-3 were 0.76, 0.67, and 0.51 for DPP4is, SGLT2is, and GLP1RAs, respectively. Jensen et al. (24) also provided an analysis in which patients were stratified according to cardiovascular risk. GLP1RAs in combination with metformin protected both high- and low-risk patients. In contrast, regimens combining metformin with DPP4is or SGLT2is decreased risk of MACE-3 only in low-risk, but not high-risk, patients (24). Taken at face value, these real-world data raise questions about recent recommendations to prescribe monotherapy with SGLT2is to patients at high risk for major adverse cardiovascular outcomes (see below) (55-58). We have cited this Danish study because it is exemplary in many respects. Nevertheless, it is important to acknowledge that the Danish population may not fully represent the experiences of diverse populations living in different socioeconomic situations and with different health insurance.

Apart from the CAROLINA trial with linagliptin, recent CVOTs for diabetes drugs were conducted as placebo-controlled trials (Table 4). Strictly interpreted, these trials demonstrated that empagliflozin, canagliflozin, liraglutide, semaglutide, and dulaglutide were superior to placebo when prescribed to highcardiovascular-risk T2D patients with inadequate metabolic control (mean HbA1c levels, $8.0 \%-8.7 \%$ ). In the absence of active comparators, it is impossible to draw rigorous conclusions about counterfactual scenarios in which patients would have been treated with other drugs to achieve comparable levels of HbA1c and blood pressure. Indeed, a recent mediation analysis of the LEADER trial of liraglutide suggests that approximately $83 \%$ of MACE-3 risk reduction would have been eliminated if $\mathrm{HbA1c}$ levels were equalized between the liraglutide and placebo arms (53).

\section{Current state of the art}

Treatment of T2D is challenging for both clinicians and patients. Pharmacotherapy aims to prevent or delay occurrence of "hard" clinical endpoints such as blindness, end-stage kidney disease, and amputations. A drug's safety profile must be balanced against its glucose-lowering efficacy. In designing a therapeutic strategy, physicians define a therapeutic target for $\mathrm{HbA1c}$ and decide which drugs to use and in what order to use them. The American Diabetes Association (ADA) and the European Association for the Study of Diabetes (EASD) have jointly formulated therapeutic guidelines, which recommend individualizing HbA1c targets based on multiple considerations: (a) expected longevity; (b) presence of diabetic complications; and (c) patient preferences (55). Guidelines suggest that targeting an HbA1c of $7.0 \%$ may be appropriate early in the course of T2D in an otherwise healthy patient, but targeting $8.0 \%-8.5 \%$ might be appropriate for a patient with limited life expectancy. ADA/EASD guidelines recommend metformin as first-line therapy for HbA1c-lowering in T2D and suggest four 


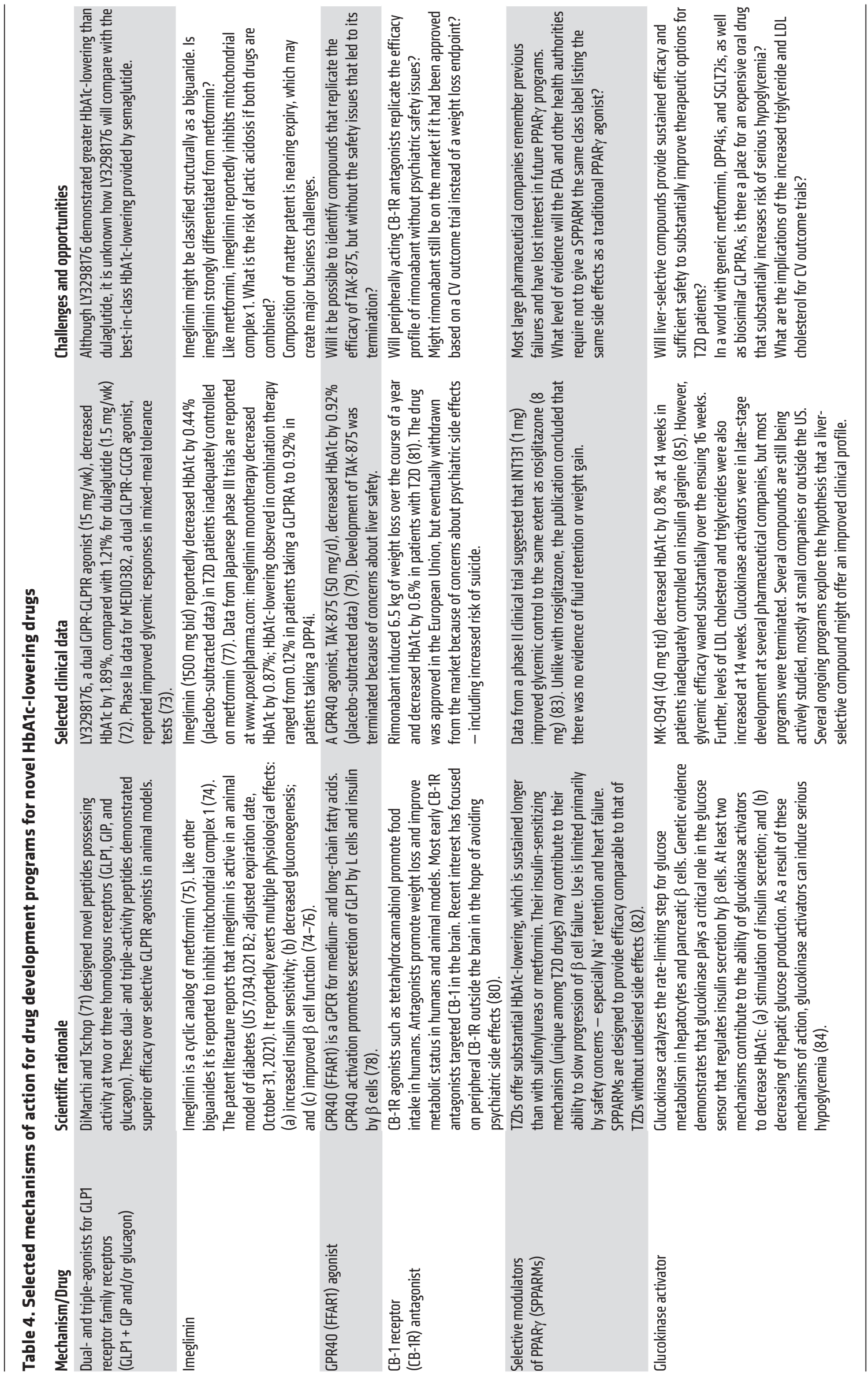




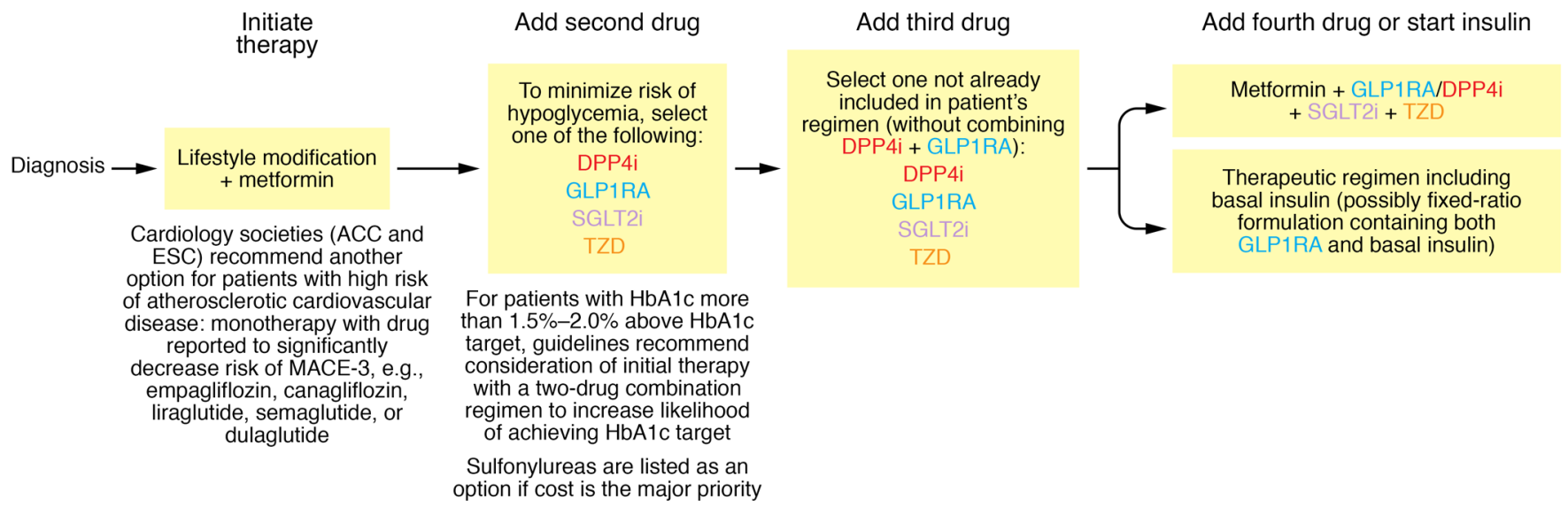

Figure 2. Overview of ADA/EASD guidelines. The ADA and EASD provided detailed guidance about pharmacological approaches to treat hyperglycemia in diabetic patients (55). The figure illustrates a simplified version of these guidelines. Initiation of therapy: Guidelines advocate simultaneous initiation of metformin and lifestyle modification (i.e., promoting weight loss in patients who are overweight or obese). Guidelines also suggest consideration of an option to initiate two-drug combination therapy if the patient's HbA1c is more than $1.5 \%-2.0 \%$ above the HbA1c target (e.g., patients with HbA1c $>8.5 \%-9.0 \%$ if the HbA1c target is 7.0\%). Addition of second drug: Many patients experience secondary failure as T2D progresses, and require addition of a second drug. ADA/EASD guidelines recommend one of four drug classes for second-line therapy: DPP4is, GLP1RAs, SGLT2is, or TZDs. Low-cost generic sulfonylureas represent a fifth option if cost considerations are the major concern. Third-and fourth-line drug. If necessary, three- and four-drug combinations can be constructed with additional drugs from among DPP4is, GLP1RAs, SGLT2is, and TZDs in combination with metformin. Many patients will eventually experience severe $\beta$ cell failure and transition to insulin-dependent physiology requiring therapy with basal insulin. With one important exception, guidance from the ACC, ESC, and AHA resembles that from the ADA/EASD (56-58). Both the ACC and the ESC have advocated for monotherapy with either GLP1RAs or SGLT2is in patients at high risk for atherosclerotic heart disease. However, it is important to emphasize that there is relatively little evidence to support this recommendation as more than $80 \%$ of patients in CVOTs with SGLT2is or GLP1RAs were receiving metformin as part of their therapeutic regimens. The ADA/EASD guidelines provide an inclusive list of options allowing physicians and patients considerable freedom to select whichever drug(s) they prefer. Many physicians may want simpler guidelines offering fewer options, such as we propose in Figure 3.

principal options for drugs to be added in patients not achieving their therapeutic target: DPP4is, GLP1RAs, SGLT2is, or TZDs (Figure 2). Sulfonylureas are offered as another option if cost is the major issue. GLP1RAs and SGLT2is are assigned priority in patients with compelling need to lose weight and patients with established atherosclerotic cardiovascular disease (ASCVD) or high risk of developing ASCVD. Guidelines consider initiating therapy with a two-drug combination (e.g., metformin+DPP4i) with the intent of sustaining longer-duration effectiveness (55, 59). Insulin is envisioned for patients who are inadequately controlled despite receiving three or four of the aforementioned drugs (55). This strategy is based on adding as few drugs as possible to achieve patients' individualized HbA1c targets, and prescribing additional drugs only when patients fail to achieve those targets. Cardiology organizations including the American College of Cardiology (ACC), the European Society of Cardiology (ESC), and the American Heart Association (AHA) provide guidelines with similar recommendations (56-58) - albeit adding an option of first-line monotherapy with GLP1RAs or SGLT2is in patients with established high risk for ASCVD.

DeFronzo and colleagues $(3,60)$ proposed a more aggressive therapeutic strategy, prescribing multiple drugs early in the course of T2D to achieve lower HbA1c levels - thereby decreasing risk of microvascular complications and possibly preserving $\beta$ cell function. DeFronzo's regimen includes three drugs: (a) metformin to decrease hepatic glucose production; (b) GLP1RA to promote weight loss and enhance insulin secretion; and (c) pioglitazone as an insulin sensitizer that indirectly preserves $\beta$ cell function. This intensive therapeutic regimen enabled newly diagnosed patients to achieve a mean $\mathrm{HbA1c}$ of $5.95 \%$; patients receiving conventional therapy achieved HbA1c of $6.5 \%$ (60). Patients receiving initial three-drug therapy experienced 7.5 -fold lower risk of hypoglycemia. This intensive therapeutic strategy offers substantial potential to improve outcomes for $\mathrm{T} 2 \mathrm{D}$ patients.

ADA/EASD guidelines maximize freedom of choice by offering five options for second-line therapy (DPP4is, SGLT2is, GLPRAs, TZDs, and sulfonylureas). Nevertheless, because some physicians and patients may welcome a shorter list of options, we offer an abbreviated version of the ADA/EASD guidelines assigning priority to drugs that provide particularly attractive options for the most common scenarios (Figure 3). Our recommended prioritization of options is based on assigning high priority to several objectives: (a) driving toward ambitious glycemic targets to minimize risk of microvascular complications so long as this can be accomplished with non-hypoglycemia-inducing drugs; (b) minimizing risks of safety and tolerability issues; and (c) taking a longterm perspective focused on a time when generic SGLT2is and DPP4is will be available.

Available literature suggests a few principles to guide HbA1c-lowering pharmacotherapy: (a) To minimize risk of microvascular complications, physicians and patients should strive for the lowest level of HbA1c that can be achieved safely. ACCORD confirmed that mean HbA1c levels of $6.5 \%$ slowed progression of diabetic retinopathy compared with HbA1c levels of 7.5\% (12). Combinations of metformin with GLP1RAs, DPP4is, or SGLT2is enable patients to achieve lower HbA1c targets without increased risk of hypoglycemia associated with sulfonylureas or insulin. Furthermore, avoiding sulfonylureas may delay secondary fail- 


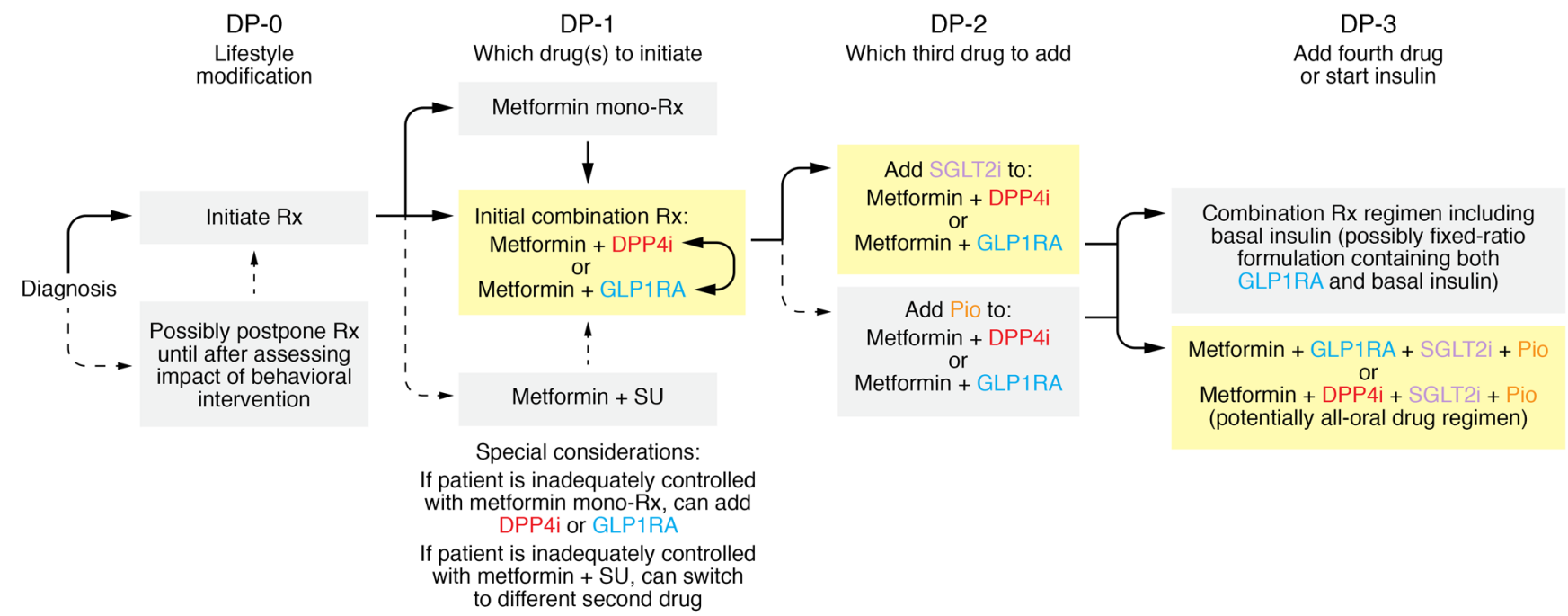

Figure 3. Algorithm to guide selection of HbA1c-lowering drugs for T2D patients. DP-O: lifestyle modification. Although lifestyle modification may sufficiently improve glucose levels to avoid the need for drugs, pharmacotherapy and lifestyle modification are most often initiated simultaneously. DP-1: which drug(s) to initiate. As recommended by the ADA/EASD, metformin should ordinarily serve as the foundation for pharmacotherapy. Nevertheless, the fact that patients will often benefit from intensive therapy early in the natural history of T2D (60) favors the ADA/EASD guidelines' option to initiate two drugs simultaneously. DPP4is and GLP1RAs have favorable safety profiles without increasing risk of serious hypoglycemia in this setting. Driving to lower HbA1c levels diminishes risk of microvascular complications (12). Although addition of a second drug increases cost, this will become less of an issue after DPP4is become generic. DP-2: which third drug to add? Many patients experience secondary failure, requiring intensification of therapy. Achievement of ambitious HbA1c targets favors the objective of minimizing risk of microvascular complications $(12,60)$. SGLT2is are an attractive component of three-drug regimens (e.g., metformin+DPP4i+SGLT2i or metformin+GLP1RA+SGLT2i), especially because of lowering of blood pressure, weight loss, renoprotection, and reported cardiovascular benefits. The "all-oral" three-drug option is available as convenient fixed-dose combination tablets (metformin+DPP4i+SGLT2i), which will be more affordable when DPP4is and SGLT2is become generic. We deprioritize pioglitazone despite its attractive efficacy profile (117) because of safety concerns with TZDs (17). If secondary failure occurs in patients receiving metformin+DPP4i, the regimen can be intensified by substitution of a GLP1RA in place of a DPP4i. DP-3: whether to initiate insulin. Insulin is often the best option for patients experiencing secondary failure on a three-drug regimen. Nevertheless, some patients may be manageable with a four-drug regimen that does not include hypoglycemia-inducing drugs such as insulin. Metformin+DPP4i+SGLT2i+pioglitazone and metformin+GLP1RA+SGLT2i+pioglitazone stand out as the most attractive among these regimens.

ure (16). (b) Intensive HbA1c-lowering early in the disease's natural history provides long-term benefit even if ambitious HbA1c targets are not sustained permanently $(5,8)$. Indeed, HbA1clowering pharmacotherapy contributes to decreasing cardiovascular risk, especially if sustained over long periods of time. (c) While glycemic control is a critical element of therapeutic regimens for T2D, other elements are also important, including lifestyle and cardiovascular medications. Based on these guiding principles, we propose the following strategy to guide prescription of HbA1clowering drugs for patients with T2D. This strategy focuses on specific decision points (DPs) arising during a patient's journey through the natural history of T2D (Figure 3).

DP-O: lifestyle modification. Most authorities recommend initiating lifestyle modification at the time T2D is diagnosed - especially weight loss in obese/overweight patients. Changes in eating behaviors and exercise habits can substantially improve metabolic control. Although postponing use of HbA1c-lowering drugs until after observing the impact of behavioral interventions offers potential (with low probability) to avoid pharmacotherapy, pharmacotherapy is most often initiated in parallel with behavioral interventions. Although outside the scope of this Review, lifestyle modifications offer enormous benefits for patients who achieve sustainable weight loss.

DP-1: which drug(s) to select as initial pharmacotherapy. ADA/ EASD guidelines recommend metformin as first-line HbA1c- lowering therapy in T2D (55). Historically, HbA1c of $7.0 \%$ was selected as a therapeutic target $(4,6)$. While available evidence suggests that lower HbA1c levels would further decrease the risk of microvascular complications, the $7.0 \%$ target was selected to mitigate risk of serious hypoglycemia in insulin- or sulfonylureatreated patients. GLP1RAs, DPP4is, SGLT2is, and TZDs do not intrinsically increase hypoglycemia risk; this creates an opportunity to achieve lower HbA1c and further decrease the risk of microvascular complications (12). We, therefore, favor ADA/ EASD guidelines' option of initiating therapy with two-drug combinations. Combinations of metformin+DPP4i or metformin+GLP1RA can achieve lower HbA1c levels than metformin monotherapy without increasing risk of serious hypoglycemia. Convenient fixed-dose combinations of metformin+DPP4i are available, and will likely become inexpensive when DPP4is become generic in the relatively near future. Combining metformin with a GLP1RA is particularly attractive. For example, semaglutide offers "normalized" HbA1c-lowering of about $2.1 \%$ combined with placebo-subtracted weight loss of about $5 \mathrm{~kg}$ and an approximately $26 \%$ decrease in risk of MACE-3 $(26,47)$. Long-term follow-up studies suggest that intensive HbA1c-lowering offers long-term clinical benefit even if improvement in $\mathrm{HbA1c}$ cannot be sustained forever $(5,8)$. We deprioritize combination therapy with metformin+sulfonylureas because of increased risk of life-threatening hypoglycemia. Furthermore, real-world data suggest higher risks of adverse 
cardiovascular events - even in patients with low cardiovascular risk (24). We also deprioritize initial therapy with metformin+SGLT2i because of increased risks of genitourinary infections and other safety issues $(41,61)$.

Many patients receive metformin monotherapy rather than initial two-drug combinations. If a monotherapy patient does not achieve the therapeutic target, a second drug (preferably a DPP4i or GLP1RA) can be added subsequently. If a patient does not achieve the therapeutic target in response to metformin+DPP4i, therapy can be intensified by substitution of a GLP1RA for the DPP4i (25).

The ACC (58) and ESC (57) advocate monotherapy with GLP1RAs or SGLT2is as options for first-line therapy. While these options may be appropriate for metformin-intolerant patients, only limited data exist to assess cardiovascular outcomes in patients receiving long-term monotherapy with SGLT2is or GLP1RAs. Approximately $80 \%$ of patients in CVOTs received SGLT2is or GLP1RAs in combination with metformin. These considerations support the ADA/EASD's recommendation to build on a foundation of metformin as first-line therapy even in patients with a history of or high risk for ASCVD. Nevertheless, available data support metformin+GLP1RA combinations as initial therapy in such high-risk patients. Metformin+SGLT2i combinations have unique value in patients with heart failure $(37-40,62,63)$. In the absence of a history of heart failure, we believe that SGLTis should be reserved as third-line options because of safety/tolerability concerns with this class.

DP-2: add-on drugs for patients inadequately controlled on metformin plus GLP1RA or DPP 4i? Because T2D is a progressive disease, many patients will experience secondary failure after having responded adequately to their therapeutic regimens. Addition of a SGLT2i will often be the best option as a third-line drug: e.g., metformin+DPP4i+SGLT2i or metformin+GLP1RA+SGLT2i. The "all-oral" option is facilitated by convenient fixed-dose combination formulations containing metformin+DPP4i+SGLT2i in the same pill. When both DPP4is and SGLT2is become generic, this three-drug combination (metformin+DPP4i+SGLT2i) will be relatively inexpensive. Although we deprioritize sulfonylureas because of safety concerns and accelerated secondary failure, they continue to be used because of long-standing historical experience and generic pricing. If sulfonylurea-treated patients do not meet their glycemic target, physicians should consider replacing the sulfonylurea with another drug such as DPP4i, GLP1RA, SGLT2i, or possibly generic pioglitazone. Discontinuation of the sulfonylurea will decrease risk of hypoglycemia and avoid the risk of sulfonylurea-associated acceleration of secondary failure (16). Although pioglitazone was reported to decrease risk of MACE-3 (54), we deprioritize pioglitazone because of risks of congestive heart failure and bone fracture. While adverse effects of TZDs on bone health are observed in both sexes (17), women are particularly vulnerable.

DP-3: whether to add insulin to the therapeutic regimen. Some patients do not achieve therapeutic targets on three-drug regimens. Although four-drug regimens are possible (e.g., metformin+DPP4i+SGLT2i+pioglitazone), many physicians and patients introduce insulin at this stage. Although detailed discussion is outside the scope of this Review, insulin therapy can be extremely effective at achieving glycemic control in this setting. Furthermore, fixed-ratio formulations are available including both basal insulin and GLP1RA. Both components offer substantial HbA1c-lowering; the GLP1RA component counteracts the weight gain that frequently accompanies insulin therapy $(64,65)$. The principal downsides of insulin relate to risk of serious hypoglycemia and therapeutic complexity. Nevertheless, insulin may be the only option that can provide acceptable glycemic control in some patients at this late stage of T2D.

\section{The future}

Impact of future losses of marketing exclusivity. Outcomes of pharmaceutical research and development (R\&D) are notoriously difficult to predict. Very few early-stage R\&D projects yield approved drugs; not all approved drugs achieve commercial success. Nevertheless, two predictions can be made with confidence. Patents will expire. Marketing exclusivity will end. It is not always possible to predict exact dates for these two events. Legal complexities create uncertainty about which patents will survive legal challenge and how long regulators will extend marketing exclusivity beyond patent expiry. Nevertheless, generic versions of DPP4is and SGLT2is will become available relatively soon. For example, the US patent on composition of matter for dapagliflozin expired in October 2020, and that for saxagliptin expires in July 2023 (66). Patent expiration is a first step toward availability of generic drugs. Availability of low-price generic SGLT2is and DPP4is will provide clinically attractive, low-cost alternatives to generic sulfonylureas (67). Availability of generic DPP4is and SGLT2is will greatly increase patient access and meaningfully decrease health care inequities (67). Because peptide drugs are regulated differently, biosimilar GLP1RAs may not have the same transformational impact on affordability.

Precision medicine: scientific foundation for individualizing therapy. At present, physicians generally select HbA1c-lowering drugs based on mean responses of average patients. The ADA and EASD noted, "in head-to-head studies, any differential effects of [antidiabetic drugs] ... are small. So ... properties such as dosing frequency, side-effect profiles, cost, and other benefits often guide their selection" (68). In contrast to small differences in mean effects of different therapies, there is wide variation of individual responses to the same drug. To implement the aspirational objective of individualizing choices of drugs, diabetes researchers must build a strong scientific foundation based on rigorous data to predict individual responses. Metformin illustrates the potential of pharmacogenomics to predict drug responses. Because metformin is positively charged at physiological $\mathrm{pH}$, membrane transporters are required for transport across biological membranes. A substantial body of research has identified genetic variants in transporter genes associated with altered function of critical transporters required for pharmacological responses to metformin. These variants alter absorption from the gastrointestinal tract, renal handling, or entry into hepatocytes (69). Variants in other genes are associated with altered pharmacodynamic responses. An intronic variant (rs8192675) was identified in SLC2A2 (encoding GLUT2 glucose transporters) that exerts a cis-acting effect to regulate GLUT2 mRNA expression (70). The minor allele of rs8192675 was associated with enhanced metformin-induced HbA1c-lower- 
ing effects. GLUT2 is expressed in several cell types with critical roles in metabolic physiology, and facilitates glucose entry into pancreatic $\beta$ cells and hepatocytes and glucose efflux through the basolateral membranes of renal proximal tubule epithelial cells and intestinal epithelium. Pioneering research with metformin illustrates the potential to build a similar scientific foundation for other HbA1c-lowering drugs - thereby transforming therapeutic strategy by selecting drugs likely to be most effective at lowering HbA1c safely in each individual patient.

Potential for innovative drugs with novel mechanisms. The first two decades of the 21st century have been a golden age for pharmaceutical R\&D, with introduction of three important new classes of diabetes drugs - two of which reported improved cardiovascular outcomes. When generic versions of these drugs become available, cost considerations will likely relegate expensive new T2D drugs to fourth-line therapy after metformin, DPP4is, GLP1RAs, and SGLT2is. This creates major challenges for companies to achieve business success in commercializing novel HbA1c-lowering drugs. Although some novel HbA1c-lowering mechanisms have been explored in recent years (Table 4) (71-85), some pharmaceutical companies have refocused R\&D to address high unmet medical need associated with diabetic complications such as diabetic kidney disease and nonalcoholic steatohepatitis (NASH). SGLT2is have ancillary benefits to slow progression of diabetic kidney disease $(39,40,86,87)$. GLP1RAs may slow progression of both NASH and diabetic kidney disease (88-91). In addition, pharmaceutical research continues to seek new chemical entities to decrease risk of diabetic complications by $\mathrm{HbA1c}$-independent mechanisms. Despite impressive progress over the past two decades, T2D remains one of the major causes of morbidity, mortality, and human suffering. Much work remains to be done!

\section{Acknowledgments}

ALB and SIT acknowledge research support from the American Diabetes Association (1-16-ICTS-112) and the National Institute of Diabetes and Digestive and Kidney Diseases (NIDDK) (R01DK118942; P30DK072488). ZSY is supported by a training grant from the NIDDK (T32DK098107).

Address correspondence to: Simeon I. Taylor, University of Maryland School of Medicine, HSF-III, Room 4182, 655 West Baltimore Street, Baltimore, Maryland 21201, USA. Phone: 410.706.6439; Email: staylor2@som.umaryland.edu.
1. Centers for Disease Control and Prevention. National Diabetes Statistics Report, 2020. https://www.cdc.gov/diabetes/library/features/ diabetes-stat-report.html. Updated February 11, 2020. Accessed November 18, 2020.

2. American Diabetes Association. 2. Classification and Diagnosis of Diabetes: Standards of Medical Care in Diabetes-2020. Diabetes Care. 2020;43(suppl 1):S14-S31.

3. Defronzo RA. Banting Lecture. From the triumvirate to the ominous octet: a new paradigm for the treatment of type 2 diabetes mellitus. Diabetes. 2009;58(4):773-795.

4. Diabetes Control Complications Trial Research Group, et al. The effect of intensive treatment of diabetes on the development and progression of long-term complications in insulin-dependent diabetes mellitus. $N$ EnglJ Med.1993;329(14):977-986.

5. Nathan DM, et al. Intensive diabetes treatment and cardiovascular disease in patients with type 1 diabetes. NEngl JMed. 2005;353(25):2643-2653.

6. [No authors listed]. Intensive blood-glucose control with sulphonylureas or insulin compared with conventional treatment and risk of complications in patients with type 2 diabetes (UKPDS 33). UK Prospective Diabetes Study (UKPDS) Group. Lancet. 1998;352(9131):837-853.

7. [No authors listed]. Effect of intensive bloodglucose control with metformin on complications in overweight patients with type 2 diabetes (UKPDS 34). UK Prospective Diabetes Study (UKPDS) Group. Lancet. 1998;352(9131):854-865.

8. Holman RR, et al. 10-year follow-up of intensive glucose control in type 2 diabetes. $N$ Engl J Med. 2008;359(15):1577-1589.

9. Gerstein HC, et al. Effects of intensive glucose lowering in type 2 diabetes. $N$ Engl J Med. 2008;358(24):2545-2559.

10. Bonds DE, et al. The association between symp- tomatic, severe hypoglycaemia and mortality in type 2 diabetes: retrospective epidemiological analysis of the ACCORD study. BMJ. 2010;340:b4909.

11. Calles-Escandón J, et al. Effect of intensive compared with standard glycemia treatment strategies on mortality by baseline subgroup characteristics: the Action to Control Cardiovascular Risk in Diabetes (ACCORD) trial. Diabetes Care. 2010;33(4):721-727

12. ACCORD Study Group, et al. Effects of medical therapies on retinopathy progression in type 2 diabetes. N Engl J Med. 2010;363(3):233-244.

13. ADVANCE Collaborative Group, et al. Intensive blood glucose control and vascular outcomes in patients with type 2 diabetes. $N$ Engl J Med. 2008;358(24):2560-2572.

14. Duckworth W, et al. Glucose control and vascular complications in veterans with type 2 diabetes. N Engl J Med. 2009;360(2):129-139.

15. Pyŏrälä K, et al. Cholesterol lowering with simvastatin improves prognosis of diabetic patients with coronary heart disease. A subgroup analysis of the Scandinavian Simvastatin Survival Study (4S). Diabetes Care. 1997;20(4):614-620.

16. Kahn SE, et al. Glycemic durability of rosiglitazone, metformin, or glyburide monotherapy. N Engl J Med. 2006;355(23):2427-2443.

17. Kahn SE, et al. Rosiglitazone-associated fractures in type 2 diabetes: an analysis from A Diabetes Outcome Progression Trial (ADOPT). Diabetes Care. 2008;31(5):845-851.

18. Mahaffey KW, et al. Results of a reevaluation of cardiovascular outcomes in the RECORD trial. Am Heart J. 2013;166(2):240-249.e1.

19. Lopes RD, et al. Methodology of a reevaluation of cardiovascular outcomes in the RECORD trial: study design and conduct. Am Heart J. 2013;166(2):208-216.e28.

20. Liao HW, et al. Pioglitazone and cardiovascular outcomes in patients with insulin resistance, pre-diabetes and type 2 diabetes: a systematic review and meta-analysis. BMJOpen. 2017;7(1):e013927.

21. Zhou X, et al. Factors contributing to the rising national cost of glucose-lowering medicines for diabetes during 2005-2007 and 2015-2017. Diabetes Care. 2020;43(10):2396-2402.

22. Nathan DM, et al. Rationale and design of the glycemia reduction approaches in diabetes: a comparative effectiveness study (GRADE). Diabetes Care. 2013;36(8):2254-2261.

23. DeFronzo RA, et al. Relationship of baseline HbA1c and efficacy of current glucose-lowering therapies: a meta-analysis of randomized clinical trials. Diabet Med. 2010;27(3):309-317.

24. Jensen MH, et al. Risk of major adverse cardiovascular events, severe hypoglycemia, and allcause mortality for widely used antihyperglycemic dual and triple therapies for type 2 diabetes management: a cohort study of all danish users. Diabetes Care. 2020;43(6):1209-1218.

25. Pratley R, et al. One year of liraglutide treatmen offers sustained and more effective glycaemic control and weight reduction compared with sitagliptin, both in combination with metformin in patients with type 2 diabetes: a randomised, parallel-group, open-label trial. Int J Clin Pract. 2011;65(4):397-407.

26. Taylor SI. GLP-1 receptor agonists: differentiation within the class. Lancet Diabetes Endocrinol. 2018;6(2):83-85.

27. Taylor SI, Leslie BR. Cardiovascular outcome trials of diabetes drugs: lessons learned. JClin Invest. 2018;128(3):893-896.

28. Smith RJ, et al. Evaluating the cardiovascular safety of new medications for type 2 diabetes: time to reassess? Diabetes Care. 2016;39(5):738-742.

29. Mishriky BM, et al. Do GLP-1RAs and SGLT-2is reduce cardiovascular events in black patients 
with type 2 diabetes? A systematic review and meta-analysis. Diabetes Obes Metab. 2019;21(10):2274-2283.

30. Mishriky BM, et al. Do GLP-1RAs SGLT-2is reduce cardiovascular events in women with type 2 diabetes? A systematic review meta-analysis [published online May 18, 2020]. Diabetes Metab. https://doi:10.1016/j.diabet.2020.05.002.

31. Scirica BM, et al. Saxagliptin and cardiovascular outcomes in patients with type 2 diabetes mellitus. N Engl J Med. 2013;369(14):1317-1326.

32. White WB, et al. Alogliptin after acute coronary syndrome in patients with type 2 diabetes. $N$ Engl JMed. 2013;369(14):1327-1335.

33. Green JB, et al. Effect of sitagliptin on cardiovascular outcomes in type 2 diabetes. $N$ Engl JMed. 2015;373(3):232-242.

34. Rosenstock J, et al. Effect of linagliptin vs glimepiride on major adverse cardiovascular outcomes in patients with type 2 diabetes: the CAROLINA randomized clinical trial [published online September 19, 2019]. JAMA. https: doi: 10.1001/ jama.2019.13772.

35. Cobble ME, Frederich R. Saxagliptin for the treatment of type 2 diabetes mellitus: assessing cardiovascular data. Cardiovasc Diabetol. 2012;11:6.

36. Engel SS, et al. Cardiovascular safety of sitagliptin in patients with type 2 diabetes mellitus: a pooled analysis. Cardiovasc Diabetol. 2013;12:3.

37. Zinman B, et al. Empagliflozin, cardiovascular outcomes, and mortality in type 2 diabetes. N Engl JMed. 2015;373(22):2117-2128.

38. Neal B, et al. Canagliflozin and cardiovascular and renal events in type 2 diabetes. N Engl J Med. 2017;377(21):2099.

39. Wiviott SD, et al. Dapagliflozin and cardiovascular outcomes in type 2 diabetes. $N$ Engl J Med. 2019;380(4):347-357.

40. Pratley RE, et al. The VERTIS CV trial: cardiovascular outcomes following ertugliflozin treatment in patients with type 2 diabetes mellitus and atherosclerotic cardiovascular disease. Diabetes Obes Metab. 2017;19(5):721-728.

41. Beitelshees AL, et al. Sodium-glucose cotransporter 2 inhibitors: a case study in translational research. Diabetes. 2019;68(6):1109-1120.

42. Inzucchi SE, et al. How does empagliflozin reduce cardiovascular mortality? Insights from a mediation analysis of the EMPA-REG OUTCOME Trial. Diabetes Care. 2018;41(2):356-363.

43. Cherney DZ, et al. Renal hemodynamic effect of sodium-glucose cotransporter 2 inhibition in patients with type 1 diabetes mellitus. Circulation. 2014;129(5):587-597.

44. Bilezikian JP, et al. Evaluation of bone mineral density and bone biomarkers in patients with type 2 diabetes treated with canagliflozin. J Clin Endocrinol Metab. 2016;101(1):44-51.

45. Watts NB, et al. Effects of canagliflozin on fracture risk in patients with type 2 diabetes mellitus. J Clin Endocrinol Metab. 2016;101(1):157-166.

46. Marso SP, et al. Liraglutide and cardiovascular outcomes in type 2 diabetes. $N$ Engl J Med. 2016;375(4):311-32.

47. Marso SP, et al. Semaglutide and cardiovascular outcomes in patients with type 2 diabetes. $N$ Engl JMed. 2016;375(19):1834-1844.

48. Gerstein HC, et al. Dulaglutide and cardiovas- cular outcomes in type 2 diabetes (REWIND): a double-blind, randomised placebo-controlled trial. Lancet. 2019;394(10193):121-130.

49. Gerstein HC, et al. Dulaglutide and renal outcomes in type 2 diabetes: an exploratory analysis of the REWIND randomised, placebo-controlled trial. Lancet. 2019;394(10193):131-138.

50. Hernandez AF, et al. Albiglutide and cardiovascular outcomes in patients with type 2 diabetes and cardiovascular disease (Harmony Outcomes): a double-blind, randomised placebo-controlled trial. Lancet. 2018;392(10157):1519-1529.

51. Holman RR, et al. Effects of once-weekly exenatide on cardiovascular outcomes in type 2 diabetes. N Engl J Med. 2017;377(13):1228-1239.

52. Pfeffer MA, et al. Lixisenatide in patients with type 2 diabetes and acute coronary syndrome. N Engl JMed. 2015;373(23):2247-2257.

53. Buse JB, et al. Cardiovascular risk reduction with liraglutide: an exploratory mediation analysis of the LEADER trial. Diabetes Care. 2020;43(7):1546-1552.

54. Dormandy JA, et al. Secondary prevention of macrovascular events in patients with type 2 diabetes in the PROactive Study (PROspective pioglitAzone clinical trial In macroVascular Events): a randomised controlled trial. Lancet. 2005;366(9493):1279-1289.

55. American Diabetes Association. 9. Pharmacologic Approaches to Glycemic Treatment: Standards of Medical Care in Diabetes-2020. Diabetes Care. 2020;43(suppl 1):S98-S110.

56. Arnold SV, et al. Clinical management of stable coronary artery disease in patients with type 2 diabetes mellitus: a scientific statement from the American Heart Association. Circulation. 2020;141(19):e779-e806.

57. Cosentino F, et al. 2019 ESC guidelines on diabetes, pre-diabetes, and cardiovascular diseases developed in collaboration with the EASD. Eur Heart J. 2020;41(2):255-323.

58. Das SR, et al. 2020 expert consensus decision pathway on novel therapies for cardiovascular risk reduction in patients with type 2 diabetes: a report of the american college of cardiology solution set oversight committee. J Am Coll Cardiol. 2020;76(9):1117-1145.

59. Matthews DR, et al. Glycaemic durability of an early combination therapy with vildagliptin and metformin versus sequential metformin monotherapy in newly diagnosed type 2 diabetes (VERIFY): a 5-year, multicentre, randomised, double-blind trial. Lancet. 2019;394(10208):1519-1529.

60. Abdul-Ghani MA, et al. Initial combination therapy with metformin, pioglitazone and exenatide is more effective than sequential add-on therapy in subjects with new-onset diabetes. Results from the Efficacy and Durability of Initial Combination Therapy for type 2 diabetes (EDICT): a randomized trial. Diabetes Obes Metab. 2015;17(3):268-275.

61. Puckrin R, Set al. SGLT-2 inhibitors and the risk of infections: a systematic review and metaanalysis of randomized controlled trials. Acta Diabetol. 2018;55(5):503-514.

62. McMurray JJV, et al. Dapagliflozin in patients with heart failure and reduced ejection fraction.
N Engl J Med. 2019;381(21):1995-2008.

63. Packer M, et al. Cardiovascular renal outcomes with empagliflozin in heart failure. $N$ Engl J Med. 2020;383(15):1413-1424.

64. Buse JB, et al. Contribution of liraglutide in the fixed-ratio combination of insulin degludec and liraglutide (IDegLira). Diabetes Care. 2014;37(11):2926-2933.

65. Rosenstock J, et al. Benefits of lixilan, a titratable fixed-ratio combination of insulin glargine plus lixisenatide, versus insulin glargine and lixisenatide monocomponents in type 2 diabetes inadequately controlled on oral agents: the LixiLan-O randomized trial. Diabetes Care. 2016;39(11):2026-2035.

66. Bristol Meyers Squib. Annual Report Pursuant To Section 13 Or 15(d) Ot The Securities Exchange Act Of 1934. Form 10-K. United States Securities and Exchange Commission. December 31, 2013. https://s21.q4cdn.com/104148044/ files/doc_financials/annual_reports/2013/BristolMyersSquibb_10K_20140214.pdf. Accessed January 6, 2021.

67. Taylor SI. The high cost of diabetes drugs: disparate impact on the most vulnerable patients. Diabetes Care. 2020;43(10):2330-2332.

68. Inzucchi SE, et al. Management of hyperglycemia in type 2 diabetes: a patient-centered approach. Position statement of the American Diabetes Association (ADA) and the European Association for the Study of Diabetes (EASD). Diabetes Care. 2012;35(6):1364-1379.

69. Pawlyk AC, et al. Metformin pharmacogenomics: current status and future directions. Diabetes. 2014;63(8):2590-2599.

70. Zhou K, et al. Variation in the glucose transporter gene SLC2A2 is associated with glycemic response to metformin. Nat Genet. 2016;48(9):1055-1059.

71. Capozzi ME, et al. Targeting the incretin/ glucagon system with triagonists to treat diabetes. Endocr Rev. 2018;39(5):719-738.

72. Frias JP, et al. Efficacy and safety of LY3298176, a novel dual GIP and GLP-1 receptor agonist, in patients with type 2 diabetes: a randomised, placebo-controlled and active comparator-controlled phase 2 trial. Lancet. 2018;392(10160):2180-2193.

73. Ambery P, et al. MEDI0382, a GLP-1 and glucagon receptor dual agonist, in obese or overweight patients with type 2 diabetes: a randomised, controlled, double-blind, ascending dose and phase 2a study. Lancet. 2018;391(10140):2607-2618.

74. Yaribeygi H, et al. Molecular mechanisms by which imeglimin improves glucose homeostasis. J Diabetes Res. 2020;2020:8768954.

75. Raczynska ED, et al. Biguanide antidiabetic drugs: imeglimin exhibits higher proton basicity but smaller lithium-cation basicity than metformin in vacuo. ACS Omega. 2018;3(12):17842-17852.

76. Perry RJ, et al. Imeglimin lowers glucose primarily by amplifying glucose-stimulated insulin secretion in high-fat-fed rodents. Am J Physiol Endocrinol Metab. 2016;311(2):E461-E470.

77. Fouqueray $P$, et al. The efficacy and safety of imeglimin as add-on therapy in patients with type 2 diabetes inadequately controlled 
with metformin monotherapy. Diabetes Care. 2013;36(3):565-568.

78. Burant CF. Activation of GPR40 as a therapeutic target for the treatment of type 2 diabetes. Diabetes Care. 2013;36(suppl 2):S175-S179.

79. Burant CF, et al. TAK- 875 versus placebo or glimepiride in type 2 diabetes mellitus: a phase 2, randomised, double-blind, placebo-controlled trial. Lancet. 2012;379(9824):1403-1411.

80. Cinar R, et al. The therapeutic potential of second and third generation $\mathrm{CB}_{1} \mathrm{R}$ antagonists. Pharmacol Ther. 2020;208:107477.

81. Van Gaal L, et al. Efficacy and safety of rimonabant for improvement of multiple cardiometabolic risk factors in overweight/obese patients: pooled 1-year data from the Rimonabant in Obesity (RIO) program. Diabetes Care. 2008;31(suppl 2):S229-S240.

82. DePaoli AM, et al. Can a selective PPAR modulator improve glycemic control in patients with type 2 diabetes with fewer side effects compared with pioglitazone? Diabetes Care. 2014;37(7):1918-1923.

83. Dunn FL, et al. Selective modulation of PPAR activity can lower plasma glucose without typical thiazolidinedione side-effects in patients with Type 2 diabetes. J Diabetes Complications. 2011;25(3):151-158.

84. Matschinsky FM, Wilson DF. The central role of glucokinase in glucose homeostasis: a perspective 50 years after demonstrating the presence of the enzyme in islets of langerhans. Front Physiol. 2019;10:148.

85. Meininger GE, et al. Effects of MK-0941, a novel glucokinase activator, on glycemic control in insulin-treated patients with type 2 diabetes. Diabetes Care. 2011;34(12):2560-2566.

86. Perkovic V, et al. Canagliflozin and renal outcomes in type 2 diabetes and nephropathy. NEngl J Med. 2019;380(24):2295-2306.

87. Wanner C, et al. Empagliflozin and progression of kidney disease in type 2 diabetes. $N$ Engl J Med. 2016;375(4):323-324.

88. Mann JFE, et al. Liraglutide and renal outcomes in type 2 diabetes. NEngl JMed. 2017;377(9):839-848.

89. Cherney DZI, et al. Dulaglutide and renal protection in type 2 diabetes. Lancet Diabetes Endocrinol. 2018;6(8):588-590.

90. Tuttle KR, et al. Dulaglutide versus insulin glargine in patients with type 2 diabetes and moderate-to-severe chronic kidney disease (AWARD-7): a multicentre, open-label, randomised trial. Lancet Diabetes Endocrinol. 2018;6(8):605-617.

91. Armstrong MJ, et al. Liraglutide safety and efficacy in patients with non-alcoholic steatohepatitis (LEAN): a multicentre, double-blind, randomised, placebo-controlled phase 2 study. Lancet. 2016;387(10019):679-690.

92. DeFronzo RA, Goodman AM. Efficacy of metformin in patients with non-insulin-dependent diabetes mellitus. The Multicenter Metformin Study Group. N Engl J Med. 1995;333(9):541-549.

93. Fujioka K, et al. Efficacy, dose-response relationship and safety of once-daily extendedrelease metformin (Glucophage XR) in type 2 diabetic patients with inadequate glycaemic control despite prior treatment with diet and exercise: results from two double-blind, placebo-controlled studies. Diabetes Obes Metab. 2005;7(1):28-39.

94. Ahmann AJ, et al. Efficacy and safety of once-weekly semaglutide versus exenatide er in subjects with type 2 diabetes (SUSTAIN 3): a 56-week, open-label, randomized clinical trial. Diabetes Care. 2018;41(2):258-266.

95. Drucker DJ, et al. Exenatide once weekly versus twice daily for the treatment of type 2 diabetes: a randomised, open-label, non-inferiority study. Lancet. 2008;372(9645):1240-1250.

96. Dungan KM, et al. Once-weekly dulaglutide versus once-daily liraglutide in metformin-treated patients with type 2 diabetes (AWARD-6): a randomised, open-label, phase 3, non-inferiority trial. Lancet. 2014;384(9951):1349-1357.

97. Buse JB, et al. Exenatide once weekly versus liraglutide once daily in patients with type 2 diabetes (DURATION-6): a randomised, openlabel study. Lancet. 2013;381(9861):117-124.

98. Pratley RE, et al. Once-weekly albiglutide versus once-daily liraglutide in patients with type 2 diabetes inadequately controlled on oral drugs (HARMONY 7): a randomised, open-label, multicentre, non-inferiority phase 3 study. Lancet Diabetes Endocrinol. 2014;2(4):289-297.

99. Rosenstock J, et al. Efficacy and safety of lixisenatide once daily versus exenatide twice daily in type 2 diabetes inadequately controlled on metformin: a 24-week, randomized, open-label, active-controlled study (GetGoal-X). Diabetes Care. 2013;36(10):2945-2951.

.Pavo I, et al. Effect of pioglitazone compared with metformin on glycemic control and indicators of insulin sensitivity in recently diagnosed patients with type 2 diabetes. JClin Endocrinol Metab. 2003;88(4):1637-1645.

101. Gaziano JM, et al. Effect of bromocriptine-QR (a quick-release formulation of bromocriptine mesylate) on major adverse cardiovascular events in type 2 diabetes subjects. J Am Heart
Assoc. 2012;1(5):e002279.

102.Zhou G, et al. Role of AMP-activated protein kinase in mechanism of metformin action. J Clin Invest. 2001;108(8):1167-1174.

103. Owen MR, et al. Evidence that metformin exerts its anti-diabetic effects through inhibition of complex 1 of the mitochondrial respiratory chain. Biochem J. 2000;348 Pt 3:607-614.

104. Miller RA, et al. Biguanides suppress hepatic glucagon signalling by decreasing production of cyclic AMP. Nature. 2013;494(7436):256-260.

105. Madiraju AK, et al. Metformin inhibits gluconeogenesis via a redox-dependent mechanism in vivo. Nat Med. 2018;24(9):1384-1394.

106.Day EA, et al. Metformin-induced increases in GDF15 are important for suppressing appetite and promoting weight loss. Nat Metab. 2019;1(12):1202-1208.

107. Coll AP, et al. GDF15 mediates the effects of metformin on body weight and energy balance. Nature. 2020;578(7795):444-448.

108.Stynen B, et al. Changes of cell biochemical states are revealed in protein homomeric complex dynamics. Cell. 2018;175(5):1418-1429.e9.

109. Inzucchi SE, et al. Efficacy and metabolic effects of metformin and troglitazone in type II diabetes mellitus. N Engl J Med.1998;338(13):867-872.

110. Buse JB, et al. The Primary glucose-lowering effect of metformin resides in the gut, not the circulation: results from short-term pharmacokinetic and 12-week dose-ranging studies. Diabetes Care. 2016;39(2):198-205.

111. Seino S. Physiology and pathophysiology of $\mathrm{K}$ (ATP) channels in the pancreas and cardiovascular system: a review. J Diabetes Complications. 2003;17(2 suppl):2-5.

112. Forman BM, et al. 15-Deoxy-delta 12, 14prostaglandin $\mathrm{J} 2$ is a ligand for the adipocyte determination factor PPAR gamma. Cell. 1995;83(5):803-812.

113. Samuel VT, et al. Lipid-induced insulin resistance: unravelling the mechanism. Lancet. 2010;375(9733):2267-2277.

114. Warshauer JT, et al. Effect of pioglitazone on plasma ceramides in adults with metabolic syndrome. Diabetes Metab Res Rev. 2015;31(7):734-744.

115. Müller TD, et al. Glucagon-like peptide 1 (GLP-1). Mol Metab. 2019;30:72-130.

116 . Boucher J, et al. Insulin receptor signaling in normal and insulin-resistant states. Cold Spring Harb Perspect Biol. 2014;6(1):a009191.

117. DeFronzo RA, et al. Pioglitazone: the forgotten, cost-effective cardioprotective drug for type 2 diabetes. Diab Vasc Dis Res. 2019;16(2):133-143. 